\title{
Serum markers of the extracellular matrix remodeling reflect antifibrotic therapy in bile-duct ligated rats
}

\author{
Robert Schierwagen ${ }^{1+}$, Diana J. Leeming ${ }^{2+}$, Sabine Klein ${ }^{1}$, Michaela Granzow ${ }^{1}$, Mette J. Nielsen ${ }^{2,3}$, \\ Tilman Sauerbruch ${ }^{1}$, Aleksander Krag ${ }^{3}$, Morten A. Karsdal ${ }^{2}$ and Jonel Trebicka ${ }^{1 *}$ \\ ${ }^{1}$ Department of Internal Medicine I, University of Bonn, Bonn, Germany \\ 2 Fibrosis Biology and Biomarkers, Nordic Bioscience, Herlev, Denmark \\ ${ }^{3}$ Department of Gastroenterology, Odense University Hospital, University of Southern Denmark, Odense, Denmark
}

Edited by:

Honglei Weng, University of

Heidelberg, Germany

Reviewed by:

Koichi Matsuzaki, Kansai Medical

University, Japan

Lungen Lu, Shanghai Jiao Tong

University School of Medicine, China

\section{${ }^{*}$ Correspondence:}

Jonel Trebicka, Department of

Internal Medicine I, University of

Bonn, Sigmund-Freud Str. 25,

D-53105 Bonn, Germany

e-mail:jonel.trebicka@

ukb.uni-bonn.de

tThese authors have contributed equally to this work.
Background: Progression of liver fibrosis is characterized by synthesis and degradation of extracellular matrix (ECM). Matrix-metalloproteinases (MMP) cleave collagen fibers at a specific site and thereby generate soluble fragments of ECM (neo-epitopes). The levels of these neo-epitopes might reflect the stage of liver fibrosis and may allow monitoring of anti-fibrotic therapies. Here we analyzed these neo-epitopes as read-out for a liver directed therapy with statins.

Methods: Bile duct ligation (BDL) was performed on wild type rats, which received atorvastatin $\left(15 \mathrm{mg} / \mathrm{kg}^{*} \mathrm{~d}\right)$ for 1 week starting at $1,2,3,4$ and 5 weeks after BDL (T1-T5), while controls remained untreated. Hepatic fibrosis was analyzed by immunohistochemistry and hepatic hydroxyproline content. TGF $\beta$ levels were measured by RT-PCR. Proteolytic activity of MMP-2 was examined by zymography. Levels of degradation MMP driven type I, III, IV and VI collagen degradation (C1M, C3M, C4M, and $\mathrm{C6M}$ ) and type III and IV collagen formation (PRO-C3 and P4NP7S) markers were assessed by specific ELISAs in serum probes.

Results: Serum markers of ECM neo-epitopes reflected significantly the deposition of ECM in the liver and were able to distinguish between early (T1-T3) and severe fibrosis (T4-T5). Statin treatment resulted in reduction of neo-epitope markers, especially when therapy was started in the stage of severe fibrosis (T4-T5). Furthermore, these markers correlated with hepatic expression of profibrotic cytokines TGF $\beta 1$ and TGF $\beta 2$. Formation markers of type III and IV collagen (PRO-C3 and P4NP7S) and degradation markers $\mathrm{C} 4 \mathrm{M}$ and $\mathrm{C6M}$ correlated significantly with hepatic MMP-2 activity in rats with severe fibrosis.

Conclusion: Determination of ECM remodeling turnover markers in serum allowed a distinction between mild and severe fibrosis. With respect to statin therapy, the markers may serve as read-out for efficacy of anti-fibrotic treatment.

Keywords: fibrosis, statins, markers, antifibrotic, ECM (extracellular matrix), remodeling

\section{INTRODUCTION}

Progressive liver fibrosis is a consequence of chronic hepatic inflammation from various causes (Friedman, 2003). The ultimate result is cirrhosis and end-stage liver disease, which has a huge impact on the patient's morbidity and mortality as well as medical and financial resources world-wide (Brundtland, 2002). The key-process during progression of liver fibrosis is the synthesis and deposition of extracellular matrix (ECM), which mainly consists of different collagen types. The responsible cells for scarformation in the liver are hepatic myofibroblasts, mainly deriving from hepatic stellate cells. These are activated and stimulated by the ongoing inflammation in the liver which is associated with formation of profibrotic cytokines including transforming growth factor $\beta$ (TGF $\beta$ ) (Friedman, 2001, 2008; Pinzani, 2002; Bataller and Brenner, 2005). However, it is important to emphasize that fibrosis progression is dynamic and constitutes both formation and degradation of ECM, which becomes imbalanced during liver disease (Gressner and Weiskirchen, 2006; Friedman, 2008; Parola et al., 2008). During this remodeling process endopeptidases such as matrix-metalloproteinase- 2 and -9 (MMP-2 and -9) are upregulated and are able to degrade excessive ECM (Barascuk et al., 2010). Small ECM protein fragments are released and may enter the circulation and may serve as systemic biochemical markers of hepatic ECM remodeling. The sites of degradation by specific MMPs are distinct and are known as neo-epitopes. These neo-epitopes can be measured using novel protein finger-print techniques (Karsdal et al., 2010, 2012).

Only few clinical trials have been conducted to test new anti-fibrotic drugs in patients with liver diseases, partly because reliable parameters for long-term monitoring of the therapeutic effect are lacking. Liver biopsy, as a widely accepted technique, 
is invasive and shows problems of gaining representative samples as well as the inter-observer variation (Bedossa et al., 2003). Transient elastography is non-invasive and a more and more accepted tool to assess fibrosis. Nevertheless, this technique carries restrictions related to cholestasis, obesity, severe inflammation and dialysis (Coco et al., 2007; Arena et al., 2008; Millonig et al., 2008, 2010; Koch et al., 2011; Trabut et al., 2012). Therefore, additional markers reflecting hepatic fibrotic activity and therapeutic effects of antifibrotic agents are needed.

Thus, the present study aims to validate novel serological markers of ECM remodeling (ECMR) that reflect hepatic fibrotic activity at different stages of fibrosis after bile-duct ligation in rats (I), and to investigate whether they mirror the therapeutic effect of HMG-CoA-reductase inhibitors (II) (Trebicka et al., 2010; Klein et al., 2012).

\section{MATERIALS AND METHODS \\ ANIMALS AND TREATMENT REGIMENS}

Sprague-Dawley rats with an initial body weight of 180-200 g were used. Twenty-four rats underwent BDL as previously described (Trebicka et al., 2007, 2010; Klein et al., 2012). Twentyone rats underwent BDL and were additionally treated with a specific dosage of atorvastatin (Trebicka et al., 2007, 2010; Klein et al., 2012) (15 mg/kg body weight per day). This study was authorized by the local committee for animal studies (Bezirksregierung Köln, 50.203.2-BN22, 46/05). All rats received chow and water ad libidum. Atorvastatin was administrated as described (Trebicka et al., 2007, 2010; Klein et al., 2012). The rats were daily weighed and pair-fed. The treatment regimens are shown in Figure 1. Respective BDL rats received atorvastatin chow for 1 week starting on distinct time points after BDL, 1 (T1), 2 (T2), 3 (T3), 4 (T4) or 5 (T5) weeks after BDL. BDL control rats were sacrificed at the corresponding time points after BDL.

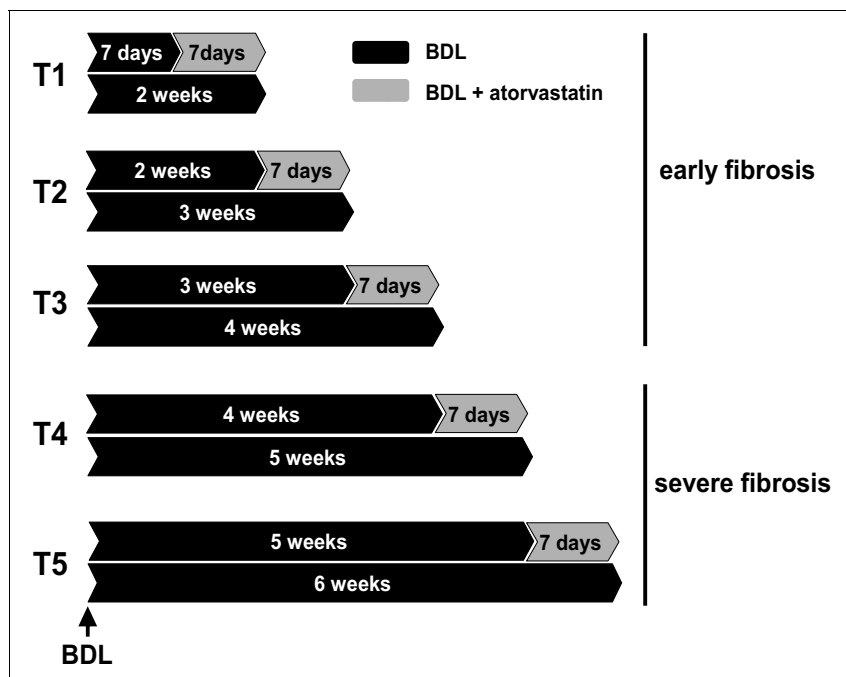

FIGURE 1 | Experimental design. Bile duct ligated (BDL) rats were used with or without atorvastatin treatment for 1 week at different times after BDL. T1 to T3 were pooled in group early fibrosis. T4-T5 were pooled in group severe fibrosis. Each group with a minimum of $n=7$.

\section{TISSUE COLLECTION AND BIOCHEMICAL ANALYSIS}

Animals were sacrificed after the described time points, blood was withdrawn to measure biochemical parameters (ALT, AP, AST, bilirubin, creatinine and $\gamma \mathrm{GT}$ ) and plasma samples were isolated using standard methods. Liver samples were cut into fragments and either snap-frozen in liquid nitrogen and stored at $-80^{\circ} \mathrm{C}$ or preserved in formaldehyde as described previously (Trebicka et al., 2007, 2008, 2010).

\section{HEPATIC HYDROXYPROLINE CONTENT}

Hepatic hydroxyproline was determined photometrically in liver hydrolysates (Trebicka et al., 2007, 2010). Segments (200 mg) of snap-frozen livers were hydrolyzed in $\mathrm{HCl}(6 \mathrm{~N})$ at $110^{\circ} \mathrm{C}$ for $16 \mathrm{~h}$ and filtered. Aliquots $(50 \mu \mathrm{l})$ from each sample were incubated with chloramine T $(2.5 \mathrm{mM})$ for $5 \mathrm{~min}$ and Ehrlich's reagent $(410 \mathrm{mM})$ for $30 \mathrm{~min}$ at $60^{\circ} \mathrm{C}$. Absorption was measured three times at $558 \mathrm{~nm}$. For determination of hydroxyproline concentration a standard curve for hydroxyproline was used. Results are expressed as $\mu \mathrm{g} / \mathrm{g}$ of wet liver tissue.

\section{SIRIUS-RED STAINING, $\alpha$ SMA-IMMUNOHISTOCHEMISTRY AND MORPHOMETRY}

Liver specimens were fixed in $10 \%$ formalin. As described previously (Trebicka et al., 2007, 2010), paraffin-embedded sections $(2-3 \mu \mathrm{m})$ were stained in $0.1 \%$ Sirius-red F3B in saturated picric acid (Chroma, Münster, Germany) to detect collagen fibers.

For immunohistochemical staining of $\alpha$-smooth muscle actin $(\alpha$ SMA $)$ sections were incubated with an mouse-anti- $\alpha$ SMA antibody (clone 1A4, Sigma-Aldrich, St. Louis, USA) diluted 1:600 in Tris-buffered saline for $60 \mathrm{~min}$. A biotinylated rabbit-anti-mouse antibody, absorbed wit rat serum (Dako, Glostrup, Denmark), was used as secondary antibody and applied subsequently diluted 1:200 for $45 \mathrm{~min}$. Streptravidin-conjugated alkaline phosphatase (1:200, $45 \mathrm{~min}$; Dako) was then attached to the complex. Sections were finally developed with new fuchsin-naphtol AS-BI (SigmaAldrich) and counterstained with hematoxyline.

Stereomicroscopic analysis was performed as described previously (Trebicka et al., 2007, 2010). On sections with a surface of $1 \mathrm{~cm}^{2}$ five hundred points were counted on a magnification of 400x. On Sirius-red staining test points were classified as connective tissue (positive staining), as well as hepatocytes, bile ducts or other structures (not stained). $\alpha$ SMA positive cells were counted on $\alpha$ SMA-immunohistological sections and characterized by their location as sinusoidal, periductular, portal/septal and others (e.g., vascular smooth muscle cells). The volume fraction of each structure was calculated as percentage of points overlaying the structure in relation to the total number of counted points.

\section{QUANTITATIVE RT-PCR}

RNA was isolated from liver segments of $30 \mathrm{mg}$ using the RNeasy kit (Qiagen, Basel, Switzerland) with DNase treatment (Promega, Wallisellen, Switzerland) followed by reverse transcription with M-MLV reverse transcriptase (Invitrogen, Basel, Switzerland) according to the manufacturers' instructions. Quantitative PCR was carried out on the ABI 7700 sequence detector (Applied Biosystems, Rotkreuz, Switzerland) with previously described sequences and accession numbers (Trebicka et al., 2010). Gene 
expression values were calculated based on $\Delta \mathrm{C}_{t}$ method (Trebicka et al., 2007, 2010; Klein et al., 2012) and normalized to expression of GAPDH. Results were calculated as $2^{-\Delta \Delta C t}$ and express the $\mathrm{x}$-fold increase of gene expression compared to sham operated rats.

\section{MMP-2 ZYMOGRAPHY}

Proteolytic activity of tissue homogenates was examined by gelatine zymography as previously published (Trebicka et al., 2010). The supernatants of liver homogenates ( $20 \mu \mathrm{g}$ of protein) were subjected to $10 \%$ SDS-PAGE using gels containing $0.3 \%$ gelatine. Proteolytic bands of 62 and $65 \mathrm{kDa}$, corresponding to the active and latent form of MMP-2 respectively were quantified by densitometry using AIDA software (Raytest, Urdorf, Switzerland).

\section{ENZYME LINKED IMMUNOSORBENT ASSAY (ELISA)}

Serological markers for MMP degradation of ECM proteins C1M (type I collagen), C3M (type III collagen), C4M (type IV collagen), C6M (type VI collagen), and formation markers PRO-C3 (type III collagen), P4NP 7S (type IV collagen) were assessed according to protocol in plasma of each rat using technical robust enzyme linked immunosorbent assays as described previously (see Table 1) (Barascuk et al., 2010; Veidal et al., 2011a,b; Leeming et al., 2011, 2012; Nielsen et al., 2013). Each marker was stratified according to time of BDL and atorvastatin treatment. The serum was obtained from previously analyzed animals (Trebicka et al., 2010).

\section{STATISTICAL ANALYSIS}

Data are presented as mean \pm standard error of the mean (SEM). Mann-Whitney- $U$-test or Wilcoxon was used for comparison between groups as appropriate. For computation of the correlations SPSS (Chicago, USA) was used. Two sided $p$-values $<0.05$ were considered statistically significant.

\section{RESULTS}

\section{SERUM LEVELS OF ECM REMODELING MARKERS MIRRORED THE QUANTITY OF HEPATIC FIBROSIS IN BDL RATS}

The serum levels of all ECMR markers of degradation and formation of ECM-proteins were negatively correlated with the hepatocyte mass (Table 2). This correlation was observed in all rats, as well as in severe fibrosis regardless of treatment (Table 2). Of note there was no correlation of any ECMR marker with creatinine and therefore these markers were not influenced by renal function (data not shown).
The deposition of total collagen, quantified by Sirius-red staining and hydroxyproline content in untreated rats correlated with all markers highly significant with ECM amount (Table 2, Figure 2).

Furthermore, the levels of the ECMR markers in all animals with different fibrosis severity (T1-T5), as well as in severe fibrosis (T4, T5) correlated with the hepatic collagen amount regardless of atorvastatin treatment (Table 2). However, these correlations were not as strong as in untreated animals, suggesting that atorvastatin treatment had an effect on the levels of these neo-epitope markers differentially to the hepatic collagen quantity determined by staining and hydroxyproline content.

The levels of collagen formation assessed serologically (PROC3 and P4NP7S), as well as of the collagen degradation markers $\mathrm{C} 3 \mathrm{M}$ and C6M were significantly lower in early fibrosis (T1-T3) compared to late stages of fibrosis $(\mathrm{T} 4, \mathrm{~T} 5)$ independent of statin treatment as shown in Table 3.

Summarizing these data, the markers reflect ECM deposition in the liver and discriminate between early and severe fibrosis.

\section{SERUM LEVELS OF ECM REMODELING MARKERS ANALYSED ON WEEKLY BASIS AFTER BDL}

We have previously shown that 1 week of atorvastatin treatment prevented ECM deposition in the early stages of fibrosis (T1-T3), but did not change the amount of ECM significantly in severe fibrosis (Trebicka et al., 2010). Interestingly, the ECRM markers provided a slightly different picture. When statin treatment was started 1 week after BDL (T2) the degradation markers for type III and IV collagen increased in serum of these animals (Figures 3B,C), whereas in the early fibrosis (T1) the levels of the degradation marker for collagen type VI (C6M) decreased significantly (Figure 3D). The levels of degradation marker type I collagen and formation markers for type III and IV were not significantly influenced in early fibrosis (Figures 3A,E,F).

In severe fibrosis (T4, T5), the levels of all neo-epitopes for degradation and formation show the same trend, since they are decreased by atorvastatin treatment (Figure 3). Especially in T5, we observed a statistical significant decrease in serum levels of C4M, C6M, PRO-C3, P4NP7S (Figures 3C-F). In the T5 group we have previously observed an effect of atorvastatin on the turnover of activated HSC and secretory activity of myofibroblasts due to induction of senescence in these cells (Trebicka et al., 2010; Klein et al., 2012).

Table 1 | Overview of used degradation and formation markers of ECM.

\begin{tabular}{lllllll}
\hline $\begin{array}{l}\text { Assay } \\
\text { name }\end{array}$ & Target & $\begin{array}{l}\text { Antibody } \\
\text { type }\end{array}$ & $\begin{array}{l}\text { Detection } \\
\text { range (ng/mL) }\end{array}$ & $\begin{array}{l}\text { Intra-assay } \\
\text { variation (\%) }\end{array}$ & $\begin{array}{l}\text { Inter-assay } \\
\text { variation (\%) }\end{array}$ & $\begin{array}{c}\text { References } \\
\text { C1M }\end{array}$ \\
\hline MMP-2/9/13 degraded type I collagen & Monoclonal & $0.83-500$ & 10.1 & 6.7 & Leeming et al., 2011 \\
C3M & MMP-9 degraded type III collagen & Monoclonal & $0.9-50$ & 4.7 & 6.5 & Barascuk et al., 2010 \\
C4M & MMP-2/9 degraded type IV collagen & Monoclonal & $0.6-100$ & 4.8 & 12.1 & Veidal et al., 2011a \\
C6M & MMP-2/9 degraded type VI collagen & Monoclonal & $0.3-250$ & 4.1 & 10.1 & Veidal et al., 2011b \\
PRO-C3 & N-terminal propeptide of type III collagen & Monoclonal & $0.9-200$ & 4.1 & 11.0 & Nielsen et al., 2013 \\
P4NP 7S & 7S domain of type IV collagen & Monoclonal & $7.9-500$ & 9.7 & 11.7 & Leeming et al., 2012
\end{tabular}


Table 2 | Correlations of hepatic histology and function with ECM markers.

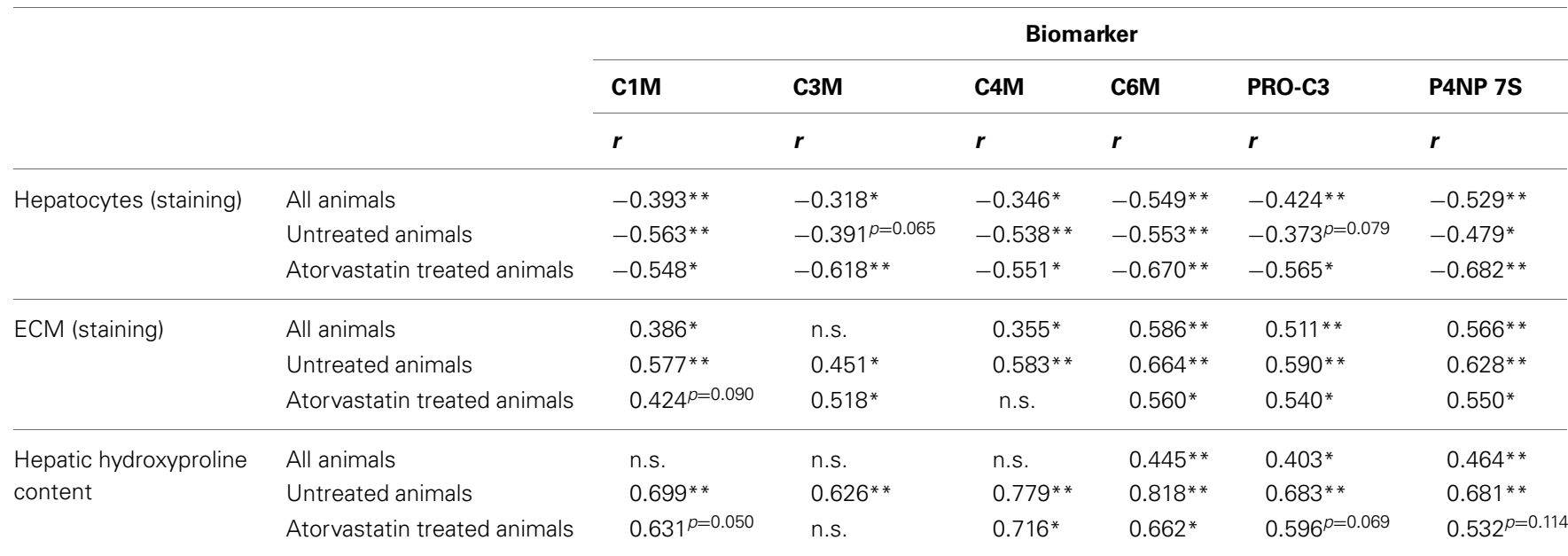

Significance of correlations were defined by non-parametric testing ( $n=$ minimum $7 /$ group) and $p<0.05$ was considered significant. Data are presented as Pearson correlation coefficient ( $r$ ) of ECM or hepatic hydroxyproline content with ECMR markers (C1M, C3M, C4M, C6M, PRO-C3 and P4NP7S). Asterisks indicate statistical significant correlations. $\left(^{*} p<0.05,{ }^{* *} p<0.01, n\right.$.s, not significant).

A

C3M and PRO-C3 correlation with hepatic hydroxyproline content

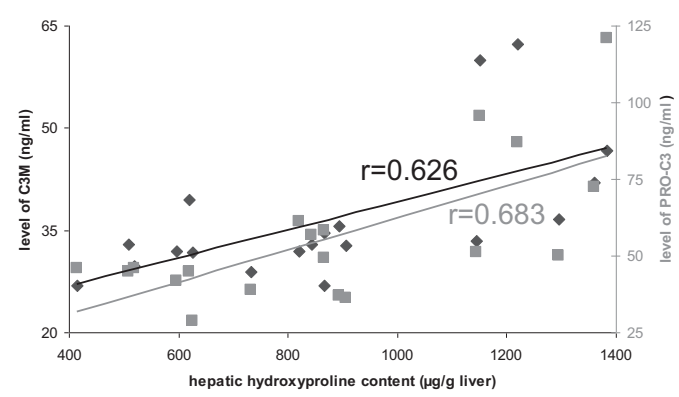

C

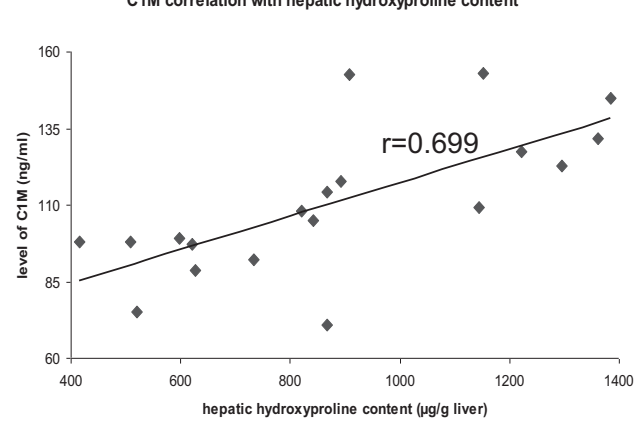

B

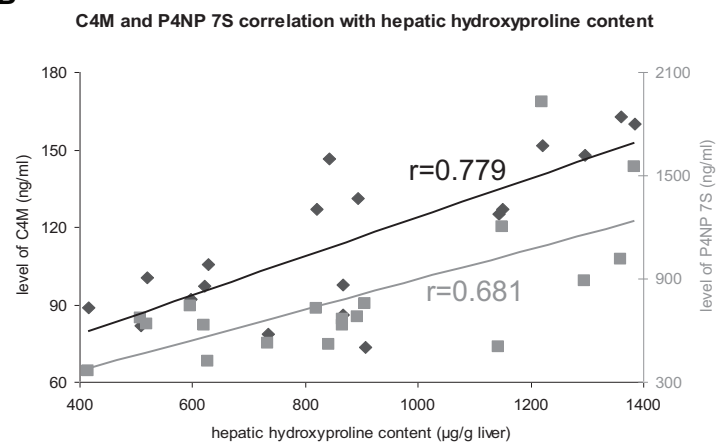

D

C6M correlation with hepatic hydroxyproline content

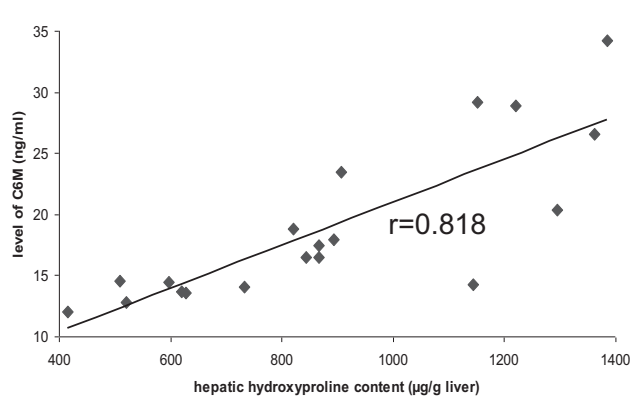

FIGURE 2 | Correlation of hepatic hydroxyproline content with ECM markers. Correlations with degradation markers shown in black, correlations with formation markers shown in gray.

\section{LEVELS OF ECM REMODELING MARKERS IN SERUM OF BDL RATS TREATED FOR 1 WEEK WITH ATORVASTATIN IN EARLY AND SEVERE FIBROSIS}

The effects of atorvastatin could be divided into basically two different types: on the one side the early effect on the prevention of activation of HSC (early stages of fibrosis) and on other side the blunting of activity and induction of senescence in myofibroblasts (severe stages of fibrosis). We, therefore, cumulatively analyzed the levels of these marker in early and severe fibrosis (Trebicka et al., 2010; Klein et al., 2012). 
Table 3 | Levels of ECM markers in blood.

\begin{tabular}{lcccccc}
\hline Group & C1M (ng/ml) & C3M (ng/ml) & C4M (ng/ml) & C6M (ng/ml) & PRO-C3 (ng/ml) & P4NP7S (ng/ml) \\
\hline T1 & $95.01 \pm 5.16$ & $34.02 \pm 2.33$ & $100.17 \pm 4.37$ & $12.32 \pm 0.60$ & $36.51 \pm 3.50$ & $535.64 \pm 55.29$ \\
T2 & $113.95 \pm 8.72$ & $37.73 \pm 2.63$ & $115.84 \pm 9.77$ & $18.12 \pm 1.37^{\mathrm{b}}$ & $50.86 \pm 2.85^{\mathrm{b}}$ & $669.76 \pm 32.23$ \\
T3 & $114.40 \pm 7.64$ & $40.15 \pm 5.26$ & $119.41 \pm 8.79$ & $15.30 \pm 1.20$ & $43.01 \pm 4.86$ & $592.13 \pm 78.00$ \\
early fibrosis & $108.01 \pm 4.62$ & $37.32 \pm 2.12$ & $111.95 \pm 4.96$ & $15.35 \pm 0.79$ & $43.73 \pm 2.45$ & $601.69 \pm 34.46$ \\
T4 & $125.25 \pm 16.76$ & $46.97 \pm 8.18$ & $127.50 \pm 17.53$ & $21.29 \pm 3.1^{\mathrm{a}}$ & $56.03 \pm 9.85$ & $932.55 \pm 131.06^{\mathrm{a}}$ \\
T5 & $117.12 \pm 8.73$ & $46.09 \pm 4.56^{\mathrm{a}}$ & $118.77 \pm 10.53$ & $21.52 \pm 2.42^{\mathrm{b}}$ & $62.29 \pm 9.15^{\mathrm{a}}$ & $989.46 \pm 140.45^{\mathrm{a}}$ \\
Severe fibrosis & $120.47 \pm 8.66$ & $46.45 \pm 4.31^{\mathrm{C}}$ & $122.37 \pm 9.57$ & $21.43 \pm 1.94^{\mathrm{d}}$ & $59.72 \pm 6.78^{\mathrm{C}}$ & $966.02 \pm 98.92^{\mathrm{d}}$
\end{tabular}

Each point in time consist of BDL rats with and without atorvastatin treatment. Points in time T1-T3 are pooled in group early fibrosis. Points in time T4-T5 are pooled in group severe fibrosis. Data are means \pm standard errors for all points of time. Letters a to $d$ indicate statistical significance. $(a, p<0.05 v s$. T1; $b, p<0.01$ vs. T1; $c, p<0.05$ vs. early fibrosis, $d, p<0.01$ vs. early fibrosis).

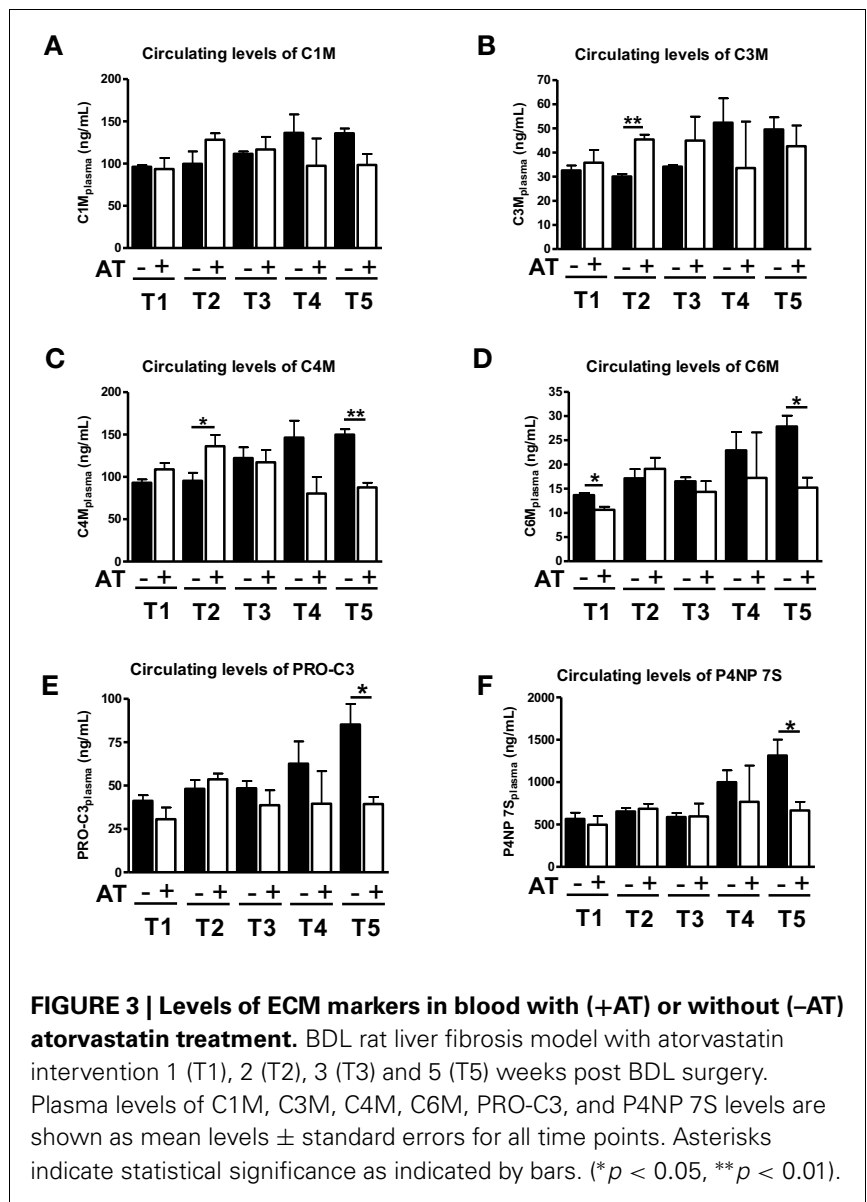

Analyzed together atorvastatin treatment for 1 week did not changed significantly the levels of ECM degradation or formation in early fibrosis $(1,2$, or 3 weeks after BDL) (Figures 4A,C-F). The only exception was the degradation neo-epitope for collagen type III, which increased after atorvastatin (Figure 4B). In a previous study these early stages showed significant lower hydroxyproline content and Sirius-red staining areas after atorvastatin treatment (Trebicka et al., 2010). By contrast, the serum levels of neo-epitopes for degradation and formation of ECM decreased after 1 week of atorvastatin treatment at late stages

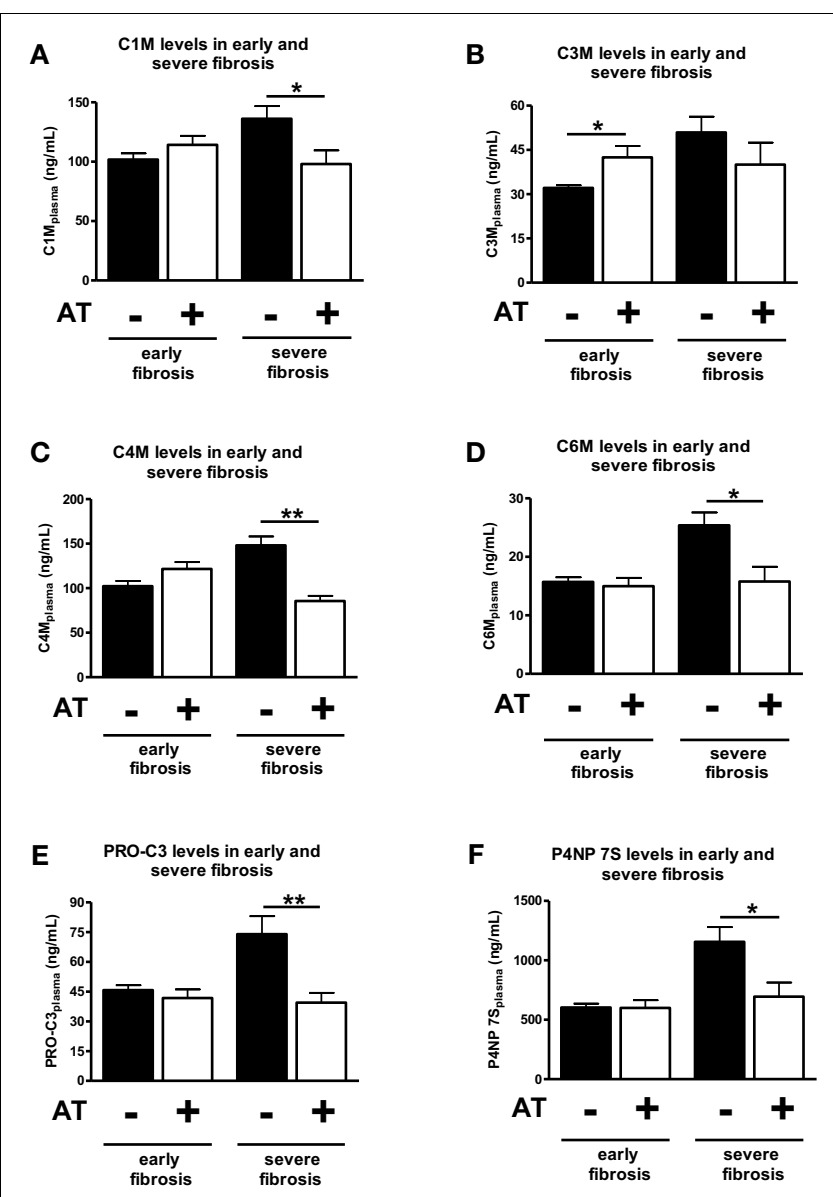

FIGURE 4 | Comparison of early and severe fibrosis for all ECM markers in atorvastatin treated or untreated animals. Different points of time were pooled into groups early fibrosis (T1-T3) and group servere fibrosis (T4 and T5) and separated by statin treated (+AT) and untreated (-AT) animals. Asterisks indicate statistical significance as indicated by bars. $\left({ }^{*} p<0.05,{ }^{* *} p<0.01\right)$.

of fibrosis (after 4 and 5 weeks of BDL) (Figure 4). These data suggest that these novel ECM degradation and formation markers might reflect the amount of fibrosis, as well as the effect of an anti-fibrotic treatment before the amount of ECM changes notably in the liver. 


\section{SERUM LEVELS OF NEO-EPITOPES OF ECM CORRELATED WITH HEPATIC EXPRESSION OF PROFIBROTIC CYTOKINES}

Hepatic mRNA levels of TGF $\beta 1$ and TGF $\beta 2$ in BDL rats were highly correlated with the serum levels of degradation markers for type I and IV collagen, as well as formation markers for type III and IV collagen, regardless of treatment (Table 4, Figures 5A,B). Interestingly, the hepatic mRNA levels of TGF $\beta 1$ and TGF $\beta 2$ showed strong correlation with almost all these neo-epitopes of ECM, when the early or the severe fibrosis group was observed separately. Similarly, strong correlations of TGF $\beta 1$ and TGF $\beta 2$ hepatic mRNA levels were observed with these markers when the treated and untreated animals were analyzed separately (Table 4; Figures 5A,B). Of note, atorvastatin treatment decreased the hepatic levels of these cytokines in severe fibrosis stages of BDL animals as shown previously (Trebicka et al., 2010), similarly to the now described levels of neo-epitopes (Figure 4).

\section{THE SERUM LEVELS OF ECM REMODELING MARKERS REFLECT HEPATIC ECM TURNOVER IN BDL INDUCED FIBROSIS}

The serum levels of the degradation marker for type IV and VI collagen, as well as formation markers for type III and IV collagen correlated strongly and significantly with hepatic MMP-2 levels measured by zymography in the rats with severe fibrosis (Figures 5C-F). Active MMP-2 and its activity calculated as the ratio of active to latent MMP-2 correlated strongly with all degradation and formation markers in severe fibrosis, pointing out that ECM markers display the turnover of ECM in liver disease (Table 5, Figures 5C-F).

\section{DISCUSSION}

The present study demonstrates that novel serum markers of ECMR reflect the dynamic process of fibrosis and may thereby used to assess effects of antifibrotic therapies.

Liver fibrosis is usually a slow progressing disease and it may take decades to develop first symptoms and complications. In the end-stage of liver disease, liver cirrhosis, the patients show many severe complications and may decompensate rapidly. At these stages the only therapeutic options are liver transplantation and/or treatment of the complications. Therefore, attenuation or even interruption of fibrosis progression is an important therapeutic goal. Therapy varies dependent on etiology of disease and during therapy—no matter which — it would be most desirable to monitor its effect on the fibrotic process.

The tissue ECMR was assessed using newly developed ELISAs, that utilize monoclonal antibodies raised against specific degradation fragments unique for ECM proteins that are involved in the development of liver fibrosis and are remodeled by disease relevant proteases. They may carry unique disease "fingerprints," why this approach is coined Protein Fingerprint Technology (Karsdal et al., 2009, 2012; Barascuk et al., 2010; Vassiliadis et al., 2011; Veidal et al., 2011a,b; Leeming et al., 2012, 2013). These markers have been validated in different models and severities of fibrosis in previous studies and compared to control non-fibrotic rats (Karsdal et al., 2009, 2012; Barascuk et al., 2010; Vassiliadis et al., 2011; Veidal et al., 2011a,b; Leeming et al., 2012, 2013).

In our BDL model we observed higher levels of these markers in early fibrosis, compared to severe fibrosis (Table 3). Moreover the levels of ECMR markers correlated very strongly with the amount of ECM, especially in the untreated BDL group. In these untreated animals, the fibrosis progression was assessed on weekly basis after BDL, as previously described (Trebicka et al., 2010). This on the one hand confirms previous findings and on the other hand validates our present findings in this rat model (Table 2).

The mechanisms of progressing fibrosis have been widely investigated in animal models, and many strategies have been published to blunt or attenuate fibrosis. However, in humans only a few trials have tested anti-fibrotic agents. One important reason for this is that animals can be treated concomitantly with initiation of hepatic injury, which makes it easier to assess the

Table 4 | Correlations of mRNA levels from profibrotic cytokines with ECM markers.

\begin{tabular}{|c|c|c|c|c|c|c|}
\hline & & \multicolumn{5}{|c|}{ Biomarker } \\
\hline & & $r$ & $r$ & $r$ & $r$ & $r$ \\
\hline & All animals & $0.382 * *$ & $0.485^{* *}$ & $0.506^{* *}$ & $0.583^{* *}$ & $0.505^{* *}$ \\
\hline \multirow[t]{3}{*}{ TGF $\beta 1$ mRNA ( $x$-fold) } & Untreated animals & $0.359^{p}=0.085$ & $0.541 * *$ & $0.420^{*}$ & $0.413^{*}$ & $0.380^{p=0.067}$ \\
\hline & Atorvastatin treated animals & $0.536^{*}$ & $0.379^{p=0.090}$ & $0.372^{p=0.097}$ & $0.687^{* *}$ & $0.687^{* *}$ \\
\hline & Severe fibrosis & $0.505^{*}$ & $0.723^{* *}$ & $0.526^{*}$ & $0.560^{*}$ & $0.604^{*}$ \\
\hline \multirow[t]{4}{*}{ TGF $\beta 2$ mRNA ( $x$-fold) } & All animals & $0.345^{*}$ & $0.353^{*}$ & $0.496^{* *}$ & $0.488^{* *}$ & $0.512 * *$ \\
\hline & Atorvastatin treated animals & $0.515^{*}$ & n.s. & $0.454^{*}$ & $0.526^{*}$ & $0.646^{* *}$ \\
\hline & Early fibrosis & $0.304^{p=0.115}$ & n.s. & $0.427^{*}$ & $0.425^{*}$ & $0.332^{p=0.085}$ \\
\hline & Severe fibrosis & n.s. & $0.556^{*}$ & $0.508^{*}$ & $0.508^{*}$ & 0.622 \\
\hline
\end{tabular}

Significance of correlations were defined by non-parametric testing ( $n=$ minimum $7 /$ group) and $p<0.05$ was considered significant. Data are presented as Pearson correlation coefficient (r) of mRNA levels of profibrotic cytokines (TGF 1 and TGF $\beta 2$ ) with respective ECMR markers (C1M, C4M, C6M, PRO-C3 and P4NP 7S). Asterisks indicate statistical significant correlations. $\left({ }^{*} p<0.05,{ }^{* *} p<0.01, n . s\right.$, not significant). 
A

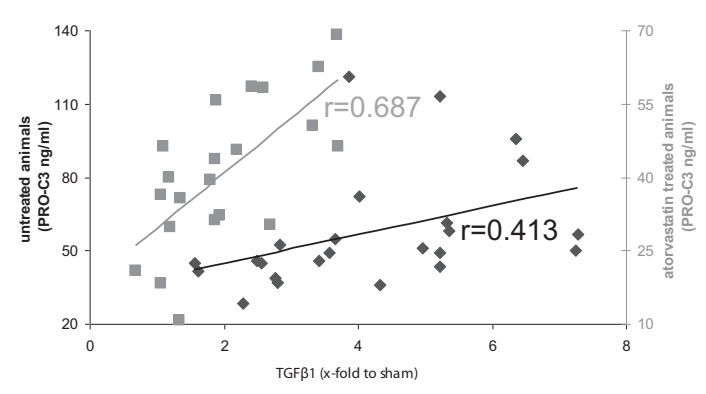

C

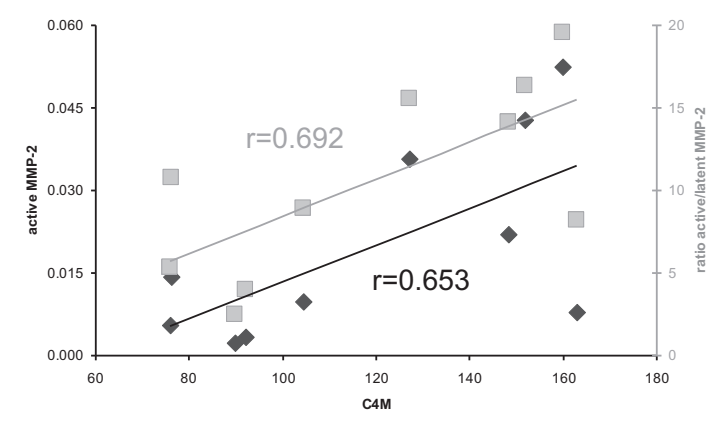

$E$

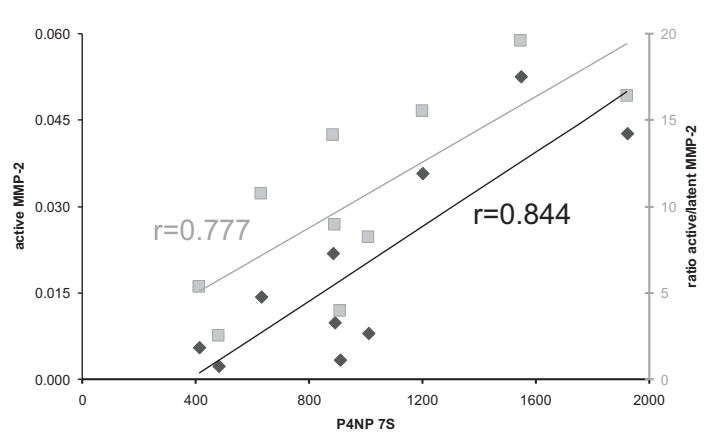

FIGURE 5 | (A,B) Correlation of formation markers with profibrotic cytokine TGF $\beta 1$ in atorvastatin treated or untreated animals. Correlations with untreated animals shown in black, correlations with atorvastatin treated
B

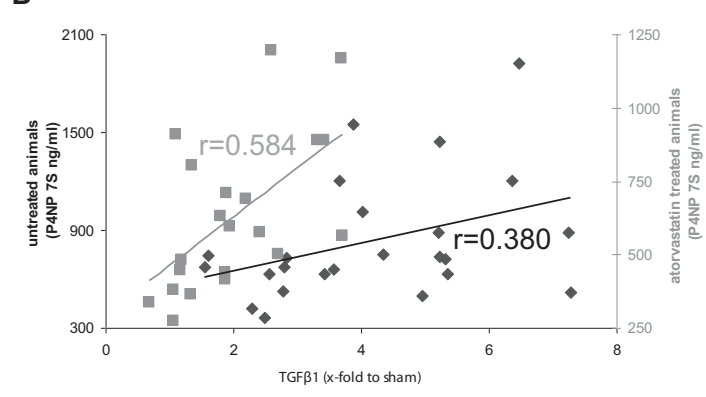

D

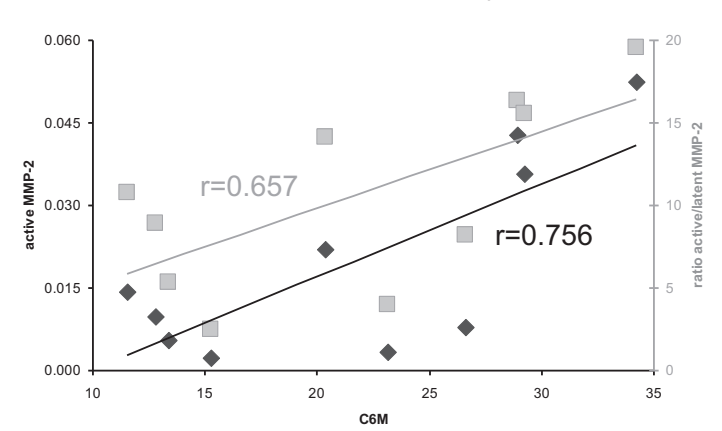

$\mathbf{F}$

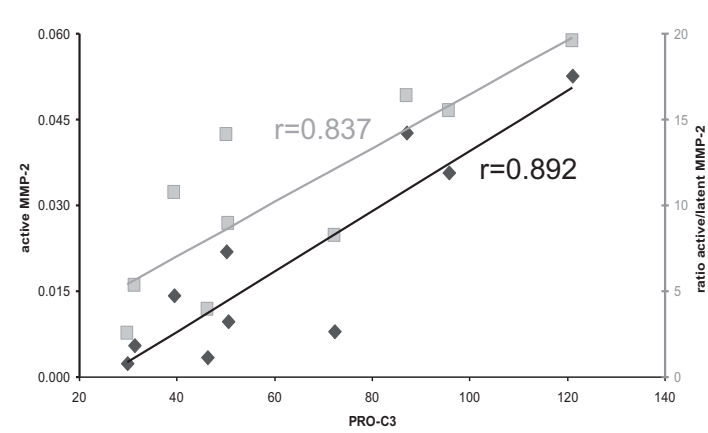

animals shown in gray. (C-F) Correlation of ECM turnover with ECM markers in severe fibrosis. Correlations with active MMP-2 shown in black, correlations of ratio active/latent shown in gray.

Table 5 | Correlation of ECM turnover with ECM markers.

\begin{tabular}{|c|c|c|c|c|c|}
\hline & & \multicolumn{4}{|c|}{ Biomarker } \\
\hline & & $r$ & $r$ & $r$ & $r$ \\
\hline MMP-2 lat & severe fibrosis & $0.576^{p=0.081}$ & $0.715^{*}$ & $0.840 * *$ & $0.835^{* *}$ \\
\hline MMP-2 akt & severe fibrosis & $0.653^{*}$ & $0.756^{*}$ & $0.892 * *$ & $0.844^{* *}$ \\
\hline MMP-2 akt/lat & severe fibrosis & $0.692^{*}$ & $0.657^{*}$ & $0.837 * *$ & $0.777 * *$ \\
\hline
\end{tabular}

Significance of correlations were defined by non-parametric testing ( $n=$ minimum $7 /$ group) and $p<0.05$ was considered significant. Data are presented as Pearson correlation coefficient (r) of MMP-2 (latent, active and ratio of active to lantent) with respective ECM markers (C4M, C6M, PRO-C3 and P4NP7S). Asterisks indicate statistical significant correlations. $\left(^{*} p<0.05,{ }^{* *} p<0.01\right.$, n.s, not significant). 
effect in the amount of fibrosis accumulated. In early fibrosis, it is easy to assess differences in the amount of fibrosis, as shown in our animal model (Trebicka et al., 2010). In severe fibrosis, the total amount of fibrosis is inadequate for the assessment of the treatment success. Atorvastatin prevents the accumulation of ECM, when it is administered in early phases of fibrosis in a BDL model of rats (Trebicka et al., 2010). Indeed, atorvastatin inhibited the activation of hepatic stellate cells in vitro the main producers of ECM in early phases of fibrosis (Klein et al., 2012).

The clinical situation is different; the patients come for treatment when fibrosis is severe and clinically evident. In these severe fibrosis the total amount of ECM does not change rapidly, therefore a long-term follow-up are needed to assess treatment effects. In our model, we mimicked this situation. In severe fibrosis ( 4 and 5 weeks after BDL) the rats were treated for 1 week with atorvastatin. The amount of ECM did not change significantly, but we observed a significant effect on the remodeling of ECM, since MMP-2 activity was blunted, the expression of profibrotic cytokines TGF $\beta 1$ and TGF $\beta 2$ were decreased (Trebicka et al., 2010; Klein et al., 2012). The serum levels of ECMR markers correlated strongly with TGF $\beta$ levels, as well as with active MMP-2 and MMP-2-activity, assessed as the ratio of active to latent MMP-2 (Tables 4, 5, Figures 5C-F). This demonstrates that the levels of these markers mirror early changes in the fibrosis progression, even before the ECM amount is changed. This is the first evidence that serum levels of these ECMR markers as read-out for antifibrotic treatment. The comparison of the accuracy of these

\section{REFERENCES}

Arena, U., Vizzutti, F., Corti, G., Ambu, S., Stasi, C., Bresci, S., et al. (2008). Acute viral hepatitis increases liver stiffness values measured by transient elastography. Hepatology 47, 380-384. doi: 10.1002/hep.22007

Barascuk, N., Veidal, S. S., Larsen, L., Larsen, D. V., Larsen, M. R., Wang, J., et al. (2010). A novel assay for extracellular matrix remodeling associated with liver fibrosis: an enzyme-linked immunosorbent assay (ELISA) for MMP-9 proteolytically revealed neo-epitope of type III collagen. Clin. Biochem. 43, 899-904. doi: 10.1016/j.clinbiochem.2010.03.012

Bataller, R., and Brenner, D. A. (2005). Liver fibrosis. J. Clin. Invest. 115, 209-218.

Bedossa, P., Dargere, D., and Paradis, V. (2003). Sampling variability of liver fibrosis in chronic hepatitis C. Hepatology 38, 1449-1457.

Brundtland, G. H. (2002). From the World Health Organization. Reducing risks to health, promoting healthy life. JAMA 288, 1974. doi: 10.1001/jama.288.16.1974

Coco, B., Olivieri, F., Maina, A. M., Ciccorossi, P., Sacco, R., Colombatto, P., et al. (2007).
Transient elastography: a new surrogate marker of liver fibrosis influenced by major changes of transaminases. J. Viral Hepat. 14, 360-369. doi: 10.1111/j.1365-2893. 2006.00811.x

Friedman, S. L. (Ed.). (2001). "The hepatic stellate cell," in Semin Liver Dis. (New York, NY: Thieme), 307-452.

Friedman, S. L. (2003). Liver fibrosisfrom bench to bedside. J. Hepatol. 38(Suppl. 1), S38-S53. doi: 10.1016/S0168-8278(02)00429-4

Friedman, S. L. (2008). Mechanisms of hepatic fibrogenesis. Gastroenterology 134, 1655-1669. doi: 10.1053/j.gastro.2008.03.003

Gressner, A. M., and Weiskirchen, R. (2006). Modern pathogenetic concepts of liver fibrosis suggest stellate cells and TGF-beta as major players and therapeutic targets. J. Cell. Mol. Med. 10, 76-99. doi: 10.1111/j.15824934.2006.tb00292.x

Karsdal, M. A., Henriksen, K., Leeming, D. J., Mitchell, P., Duffin, K., Barascuk, N., et al. (2009). Biochemical markers and the FDA Critical Path: how biomarkers may contribute to the understanding of pathophysiology and provide unique and necessary tools for

markers with other serological fibrosis markers and the investigation in other models of fibrosis should be topic of future studies.

In severe fibrosis statin treatment decreased the turnover of activated hepatic stellate cells (Trebicka et al., 2010), due to the induction of senescence in these profibrogenic cells (Klein et al., 2012). In the present study, we showed that these profibrogenic cells produce less collagen (formation markers) and the turnover of ECM is reduced as well (as shown by reduced degradation markers), which led to the observation that in the severe fibrosis statin treatment for 1 week did not decrease the static amount of total collagen in the liver assessed by hydroxyproline content and Sirius-red-staining (Trebicka et al., 2010). In conclusion, these markers reflect the release of ECM-fragments into circulation and probably mirror dynamics of fibrogenesis. Thus, they are related to disease activity and may potentially also carry prognostic information. Therefore, they should be tested for monitoring the effect of antifibrotic treatment in human diseases.

\section{ACKNOWLEDGMENTS}

The study was supported by grants from the Deutsche Forschungsgemeinschaft (SFB TRR57 project 18), the BonforStiftung (O-107.0084 and O-107.0103) and from J. \& W. HectorFoundation. The biomarker assessments were supported by the Danish science foundation ("Den Danske Forskningsfond"). The authors thank G. Hack and S. Bellinghausen for excellent technical assistance.

drug development. Biomarkers. 14, 181-202. doi: 10.1080/135475009 02777608

Karsdal, M. A., Henriksen, K., Leeming, D. J., Woodworth, T., Vassiliadis, E., and Bay-Jensen, A. C. (2010) Novel combinations of PostTranslational Modification (PTM) neo-epitopes provide tissue-specific biochemical markers-are they the cause or the consequence of the disease? Clin. Biochem. 43, 793-804. doi: 10.1016/j.clin biochem.2010.03.015

Karsdal, M. A., Nielsen, M. J., Sand, J. M., Henriksen, K., Genovese, F., Bay-Jensen, A. C., et al. (2012). Extracellular matrix remodeling: the common denominator in connective tissue diseases possibilities for evaluation and current understanding of the matrix as more than a passive architecture, but a key player in tissue failure. Assay Drug Dev. Technol. 11, 70-92. doi: 10.1089/adt.2012.474

Klein, S., Klosel, J., Schierwagen, R., Korner, C., Granzow, M., Huss, S., et al. (2012). Atorvastatin inhibits proliferation and apoptosis, but induces senescence in hepatic myofibroblasts and thereby attenuates hepatic fibrosis in rats.
Lab. Invest. 92, 1440-1450. doi: 10.1038/labinvest.2012.106

Koch, A., Horn, A., Duckers, H. Yagmur, E., Sanson, E., Bruensing, J., et al. (2011). Increased liver stiffness denotes hepatic dysfunction and mortality risk in critically ill non-cirrhotic patients at a medical ICU. Crit. Care 15, R266.

Leeming, D., He, Y., Veidal, S., Nguyen, Q., Larsen, D., Koizumi, M., et al. (2011). A novel marker for assessment of liver matrix remodeling: an enzyme-linked immunosorbent assay (ELISA) detecting a MMP generated type I collagen neo-epitope (C1M). Biomarkers 16, 616-628. doi: 10.3109/1354750X.2011.620628

Leeming, D. J., Byrjalsen, I., Jiménez., Christiansen, C., and Karsdal, M. A. (2013). Protein fingerprinting of the extracellular matrix remodelling in a rat model of liver fibrosis-a serological evaluation. Liver Int. 33, 439-447. doi: 10.1111/ liv. 12044

Leeming, D. J., Nielsen, M. J., Dai, Y., Veidal, S. S., Vassiliadis, E., Zhang, C., et al. (2012). An Enzymelinked Immunosorbent Serum Assay (ELISA) specific for the 7S domain of Collagen Type IV 
(PIVNP 7S) -a marker related to the extracellular matrix remodeling during liver fibrogenesis. Hepatol. Res. 42, 482-493. doi: 10.1111/j.1872-034X.2011.00946.X

Millonig, G., Friedrich, S., Adolf, S., Fonouni, H., Golriz, M., Mehrabi, A., et al. (2010). Liver stiffness is directly influenced by central venous pressure. J. Hepatol. 52, 206-210.

Millonig, G., Reimann, F. M., Friedrich, S., Fonouni, H., Mehrabi, A., Buchler, M. W., et al. (2008). Extrahepatic cholestasis increases liver stiffness (FibroScan) irrespective of fibrosis. Hepatology 48, 1718-1723. doi: 10.1002/hep.22577

Nielsen, M. J., Nedergaard, A. F., Sun, S., Veidal, S. S., larsen, L., Zheng, Q., et al. (2013). The neo-epitope specific PRO-C3 ELISA measures true formation of type III collagen associated with liver and muscle parameters. Am. J. Trans. Res. 5, 303-315.

Parola, M., Marra, F., and Pinzani, M. (2008). Myofibroblast - like cells and liver fibrogenesis: Emerging concepts in a rapidly moving scenario. Mol. Aspects 29, 58-66. doi: 10.1016/j.mam.2007.09.002

Pinzani, M. (2002). PDGF and signal transduction in hepatic stellate cells. Front. Biosci. 7, d1720-d1726.
Trabut, J. B., Thepot, V., Nalpas, B., Lavielle, B., Cosconea, S., Corouge, M., et al. (2012). Rapid decline of liver stiffness following alcohol withdrawal in heavy drinkers. Alcohol. Clin. Exp. Res. 36, 1407-1411. doi: 10.1111/j.15300277.2012.01737.x

Trebicka, J., Hennenberg, M., Laleman, W., Shelest, N., Biecker, E., Schepke, M., et al. (2007). Atorvastatin lowers portal pressure in cirrhotic rats by inhibition of RhoA/Rho-kinase and activation of endothelial nitric oxide synthase. Hepatology 46, 242-253. doi: 10.1002/hep. 21673

Trebicka, J., Hennenberg, M., Odenthal, M., Shir, K., Klein, S., Granzow, M., et al. (2010). Atorvastatin attenuates hepatic fibrosis in rats after bile duct ligation via decreased turnover of hepatic stellate cells. J. Hepatol. 53, 702-712. doi: 10.1016/j.jhep.2010.04.025

Trebicka, J., Leifeld, L., Hennenberg, M., Biecker, E., Eckhardt, A., Fischer, N., et al. (2008). Hemodynamic effects of urotensin II and its specific receptor antagonist palosuran in cirrhotic rats. Hepatology 47, 1264-1276. doi: 10.1002/hep. 22170

Vassiliadis, E., Larsen, D. V., Clausen, R. E., Veidal, S. S., Barascuk, N., Larsen, L., et al. (2011).
Measurement of CO3-610, a potential liver biomarker derived from matrix metalloproteinase- 9 degradation of collagen type iii, in a rat model of reversible carbontetrachloride-induced fibrosis. Biomark Insights 6, 49-58. doi: 10.4137/BMI.S6347

Veidal, S. S., Karsdal, M. A., Nawrocki, A., Larsen, M. R., Dai, Y., Zheng, Q., et al. (2011a). Assessment of proteolytic degradation of the basement membrane: a fragment of type IV collagen as a biochemical marker for liver fibrosis. Fibrogenesis Tissue Repair 4:22. doi: 10.1186/ 1755-1536-4-22

Veidal, S. S., Karsdal, M. A., Vassiliadis, E., Nawrocki, A., Larsen, M. R., Nguyen, Q. H., et al. (2011b). MMP mediated degradation of type VI collagen is highly associated with liver fibrosis-identification and validation of a novel biochemical marker assay. PLoS ONE 6:e24753. doi: 10.1371/journal.pone.0024753

Conflict of Interest Statement: Diana J. Leeming, Morten A. Karsdal, and Mette J. Nielsen are employees of Nordic Bioscience, a company engaged in the development of biochemical markers. Morten A. Karsdal is stockholder of Nordic Bioscience. The other authors declare that the research was conducted in the absence of any commercial or financial relationships that could be construed as a potential conflict of interest.

Received: 29 May 2013; paper pending published: 25 June 2013; accepted: 08 July 2013; published online: 30 July 2013. Citation: Schierwagen R, Leeming DJ, Klein S, Granzow $M$, Nielsen $M J$, Sauerbruch T, Krag A, Karlsdal MA and Trebicka J (2013) Serum markers of the extracellular matrix remodeling reflect antifibrotic therapy in bile-duct ligated rats. Front. Physiol. 4:195. doi: 10.3389/ fphys.2013.00195

This article was submitted to Frontiers in Gastrointestinal Sciences, a specialty of Frontiers in Physiology.

Copyright (c) 2013 Schierwagen, Leeming, Klein, Granzow, Nielsen, Sauerbruch, Krag, Karlsdal and Trebicka. This is an open-access article distributed under the terms of the Creative Commons Attribution License (CC BY). The use, distribution or reproduction in other forums is permitted, provided the original author(s) or licensor are credited and that the original publication in this journal is cited, in accordance with accepted academic practice. No use, distribution or reproduction is permitted which does not comply with these terms. 